\title{
The topology optimization on the bed of CNC lathe
}

\author{
Zhaokun $\mathrm{Li}^{1, \mathrm{a}}$, Yongqing Fu, b, Lijuan Shi ${ }^{1, \mathrm{c}}$, Huamei Bian ${ }^{1, \mathrm{~d}}$
}

${ }^{1}$ Engineering of mechanical and electrical department, Beijing polytechnic college, Beijing, 100042, China

${ }^{2}$ College of Mechanical Engineering, South China University of Technology Guangzhou, Guangdong, 510640, China

a2008lizhaokun@163.com, b yqfu@scut.edu.cn, c sljzch_2000@126.com, dbianhuamei0@163.com

Keywords: Topology optimization, finite element analysis, CNC lathe

Abstract: In order to improve the stiffness and dynamic characteristics of the bed for CNC lathe, based on the solid isotropic material with penalization approach, the CNC lathe mathematical model of topology optimization is developed, in which minimization of the compliance is taken as objective function and the volume is taken as constraint function; Then got the best optimal topology form of the bed through the topology optimization, new geometric model was reconstructed according to the topology form. By the analysis of primary and reconstruction models of the bed, it is found that the optimized structure not only satisfied static stiffness requirement but also satisfied dynamic requirement, which provided a scheme for the bed of CNC lathe topology optimization design.

\section{Introduction}

The bed structure of CNC lathe regarded as the important key part, whose stress, strain and natural frequencies affect the processing performance of the whole machine. Presently, the design of numerical control machine mainly depends on the experience, which exist the problems. Either the stiffness is not satisfied in order to save material or safety factor is too conservative and waste materials.

In order to simultaneously meet the requirements of static stiffness and dynamic performance, and try to save materials, it is necessary for the topology optimization design of the bed structure $[1,2]$. Topology optimization is a design method based on structure optimization, whose main idea is to pursue the optimal material distribution in a known design domain under the action of external forces and constraints.

In recent years, topology optimization study made great progress. Optimization design is extended from single target to multi-objective function, from single physical field is extended to multi physical field, from material geometric linear problem to nonlinear problem, from the static topology optimization to dynamic topology optimization [3,4]. However, applying topology optimization method to design lathe parts research is seldom, only several articles have been reported [5-8]. The research on the topology optimization of machine tool parts has started, which needs further research.

Based on the solid isotropic material with penalization approach, the CNC lathe mathematical model of topology optimization is developed, in which minimization of the compliance is taken as objective function and the volume is taken as constraint function; Then got the best optimal topology form of the bed, new geometric model was reconstructed according to the topology form. By the analysis of primary and reconstruction models of the bed, it is found that the optimized structure not only satisfied static stiffness requirement but also satisfied dynamic requirement 。

\section{Topology optimization model considering stiffness}

The system stiffness of continuum structure is used of characterize of the strain energy, when the driving load is given, the system's strain energy is smaller, the greater the system stiffness. 
Maximizing the rigidity requirement is determined by minimizing Strain Energy (SE) which is equivalent to minimizing the mean compliance $(C)$ of the structures and the formulation is defined as:

$$
\min S E \Leftrightarrow \min C=\boldsymbol{F}^{T} \boldsymbol{U}
$$

Using the SIMP approach, the relative density $x_{e}$ of material in each element is a design variable. The $N$-vector containing the design variables is denoted $\boldsymbol{x}$.

The topology optimization solving the problem of distributing a limited amount of material in the design domain such that the objective function is minimized and the volume is constrained can be expressed as:

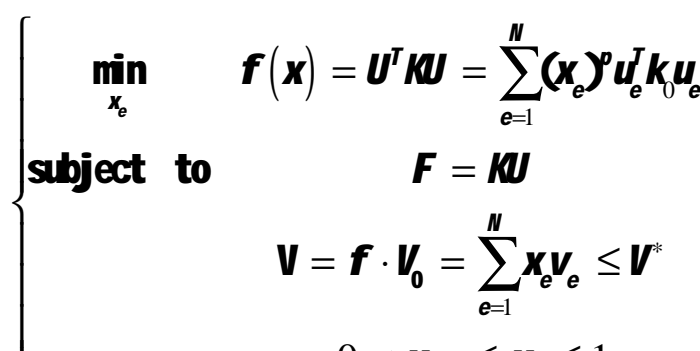

$$
\begin{aligned}
& 0<x_{\text {min }} \leq x_{e} \leq 1
\end{aligned}
$$

Where, $\boldsymbol{F}$ is external force vector and $\boldsymbol{U}$ is the displacement vector, $f$ is volume ratio, $\mathrm{K}$ is known as the tangent stiffness matrix, $\boldsymbol{V}$ is $N$-vector containing the element volume, $V_{0}$ is the upper bound on material volume and $x_{\min }$ is an $N$-vector with the minimum values of the densities $\left(x_{\min }=10^{-3}\right)$. The constitutive tensor for element $e$ with intermediate densities can be expressed as:

$$
\mathbf{C}_{i j k l}^{e}=\left(x_{e}\right)^{p} \mathbf{C}_{i j k l}^{0}
$$

The second Piola-Kirchhoff stresses are calculated:

$$
\boldsymbol{\sigma}_{i j}=x_{\mathrm{e}}{ }^{P} \boldsymbol{C}_{i j k l}^{0} \boldsymbol{\varepsilon}_{k l}
$$

where $P$ is the penalization factor (typically $P=3$ ), $C_{i j k l}^{0}$ is the constitutive tensor for solid isotropic material.

\section{The static and modal analysis of the primitive bed of numerical control lathe The static analysis of primitive bed}

The lathe bed of type HM6-L4-00 is modeled by using combining forward and reverses method. The utilized materials of the integrated lathe bed and guide is HT300, density is $7.4 \mathrm{e} 3 \mathrm{~kg} / \mathrm{m} 3$, and elastic modulus is $150 \mathrm{GPa}$, Poisson's ratio is 0.25 , the tensile strength is $300 \mathrm{MPa}$. The CNC lathe power is $5 \mathrm{~kW}$, spindle speed range is $600 \mathrm{r} / \mathrm{min}$, journal is $35 \mathrm{~mm}$, and travel Z is $300 \mathrm{~mm}$, travel X is $50 \mathrm{~mm}$. estimating the main cutting force is $5070 \mathrm{~N}$, feed force is $2535 \mathrm{~N}$ and back force is $2028 \mathrm{~N}$ and track worktable bearing load is $4000 \mathrm{~N}$. The boundary conditions of lathe bed including steady cutting force, inertia force and displacement constraints. The 3D model of finite element is as shown in Table 1.

Using the finite element analysis software Workbench, the lathe bed is loaded and simulated. Calculation result is as shown in table 1, the table 1 (b) boundary conditions; the node stress contour map is as shown in Table 1 (c), and the total displacement distribution contour map as shown in Figure $1(\mathrm{~d})$.

Table 1 (c) of bed deformation distribution shows the maximum deformation $0.28932 \mathrm{~mm}$.

Table 1 (d) shows that the maximum stress is about $42.564 \mathrm{Mpa}$, and the value of equivalent stress between $0 \sim 23.647 \mathrm{MPa}$. The blue part of the minimum stress area accounted for most of the bed. Through the calculation safety factor reached 7.05 shows that the initial bed design structure is conservative. The body structure can be improved by optimizing the structure, so as to reduce the weight of the body. 
Table1 the topological structure of primitive lathe bed

\begin{tabular}{|c|c|}
\hline (a) three-dimensional model & \\
\hline (b) Boundary conditions for static \\
analysis
\end{tabular}

\section{The modal analysis of primitive bed}

The modal of the integrated lathe bed and guide is analyzed, and then the 20 order modal expanded by using Block Lanczons method, because the influence of low order modes of structure 
vibration system is relatively large, the 6 order natural frequency and vibration model are analyzed. Table 1 (e) shows the boundary conditions for modal analysis, and table 1 (f) (h) shows the 3 order vibration models

The modal vibration diagram can bee seen that the bed is the local vibration, that is bed local structural stiffness relatively weak. The maldistribution will affect the machining accuracy of CNC lathe. The first three modal frequencies respectively are $286.34 \mathrm{~Hz}, 421.56 \mathrm{~Hz}$, and $467.03 \mathrm{~Hz}$, as far as possible to avoid the first three order vibration frequency.

\section{Topology optimization of the bed of numerical control lathe}

In order to improve the dynamic and static performance of the CNC lathe, lightweight design is carried out using topology optimization. A given design domain and boundary condition of topology optimization is shown in table 2.

Table 2 topology optimization process

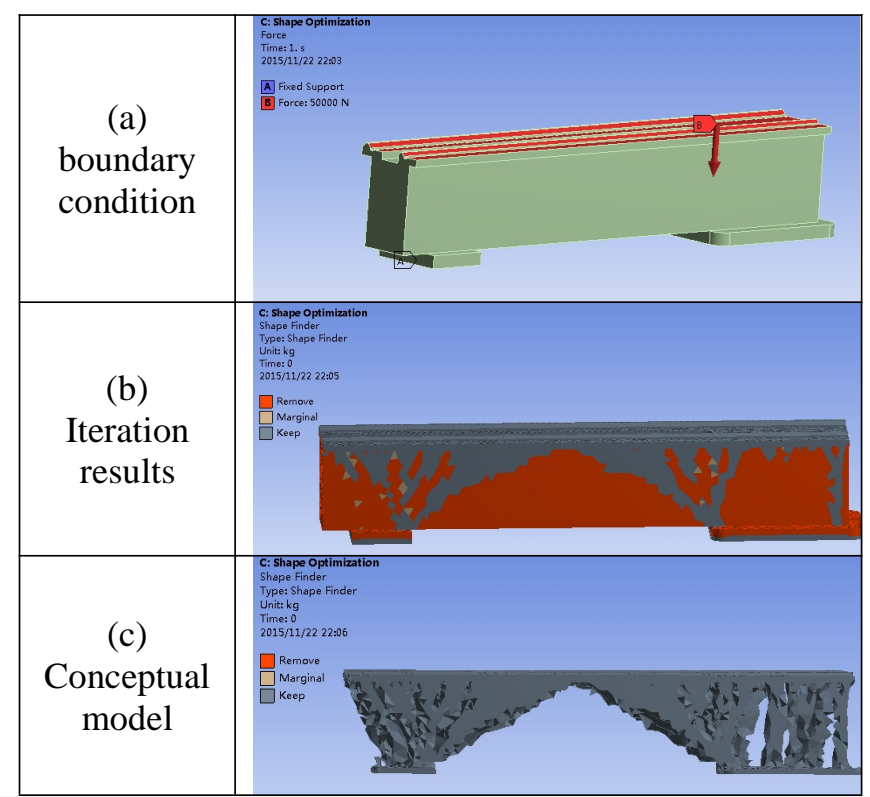

The lathe bed is optimized using finite analysis software workbench, defining the volume percentage is $50 \%$ to save material, the largest convergence tolerance is 0.0001 , and optimization iteration automatically performs 20 iterations. The topology optimization Iteration results is shown in table 2(b), the topology optimization conceptual model is shown in table 2(c).

\section{The static and modal analysis of the optimized lathe bed}

\section{the static analysis of optimized bed}

Topology optimization of lathe bed is a concept optimization design. The model needs to be reconstructed considering actual structure assembly relation, manufacturing process and actual demand. Table 2(c) shows the improved lathe bed need to reduce the size of both sides. The constructional optimized model is as shown in table 3 (a).

Using the finite element analysis software Workbench, the optimized lathe bed is loaded and the static and modal simulated in order to verify the reliability of the topology optimization. The calculation result of optimized lathe bed is as shown in table 3, the table 3 (a) boundary conditions; the node stress contour map is as shown in Table 3 (b), and the total displacement distribution contour map as shown in table 3 (c). Table 3 (b) of bed deformation distribution shows the maximum deformation $0.09 \mathrm{~mm}$. Table 3 (c) shows that the maximum stress is about $55.392 \mathrm{Mpa}$.

Compared to the primitive structure, the quality of the optimized structure reduces almost $10 \%$, maximum stress increase nearly $10 \mathrm{Mpa}$ that is far less than the tensile strength of $300 \mathrm{Mpa}$ and the biggest deformation is reduced to $0.09 \mathrm{~mm}$ that is less than $0.29 \mathrm{~mm}$ of primitive structure. 
Table 3 calculation result of optimized lathe bed

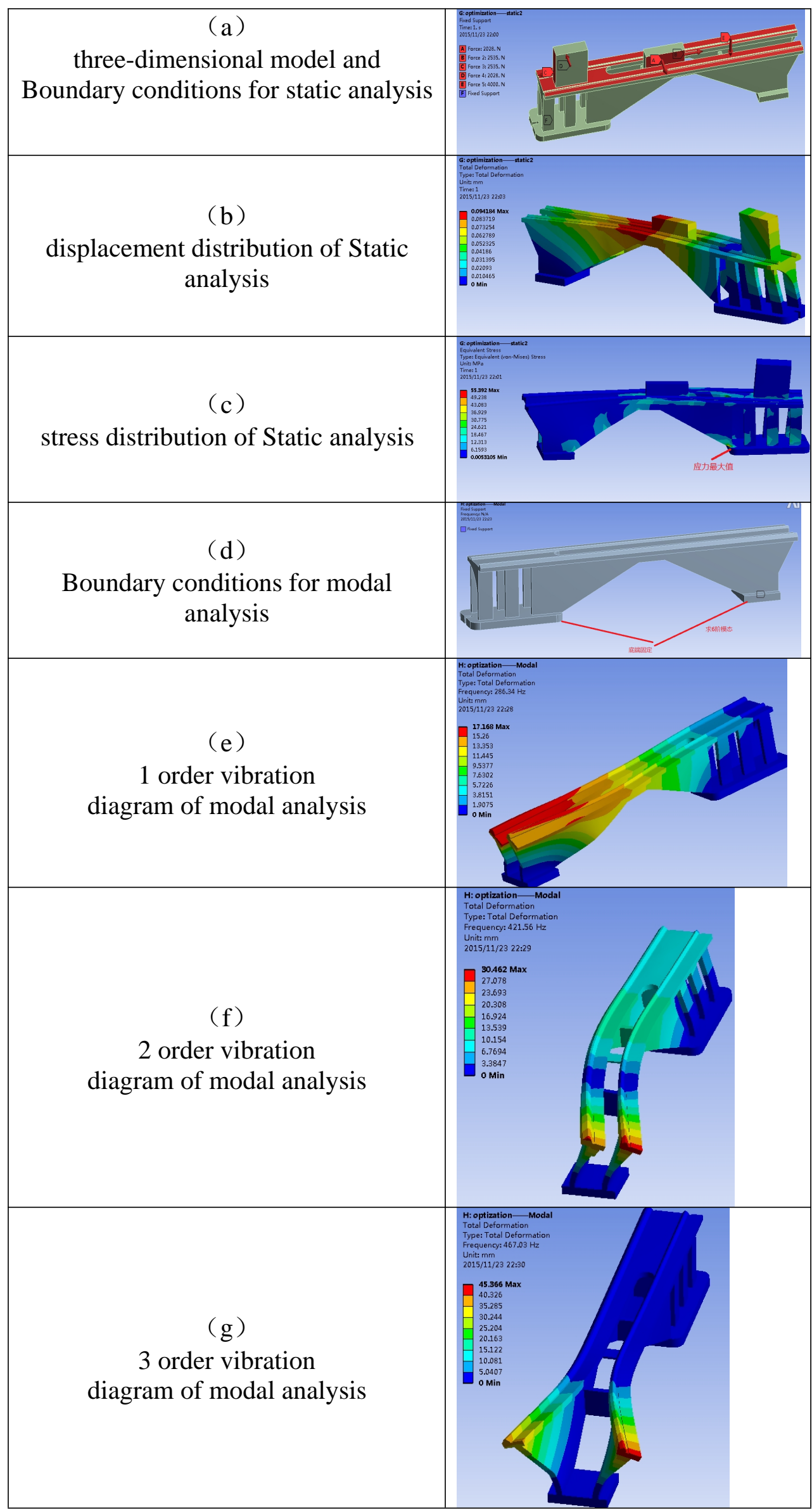


Table 4 lathe bed modal frequency comparison

\begin{tabular}{|c|c|c|c|c|}
\hline order & $\begin{array}{c}\text { Original } \\
\text { model/HZ }\end{array}$ & $\begin{array}{c}\text { Reconstruction } \\
\text { model /HZ }\end{array}$ & $\begin{array}{c}\text { Changeable } \\
\text { amount }\end{array}$ & Percentage\% \\
\hline 1 & 286.34 & 371.87 & 85.53 & 29.87 \\
\hline 2 & 421.56 & 616.88 & 195.32 & 46.33 \\
\hline 3 & 467.03 & 706.89 & 239.86 & 51.35 \\
\hline 4 & 616.02 & 927.03 & 311.01 & 50.82 \\
\hline 5 & 924.11 & 973.04 & 48.93 & 5.29 \\
\hline 6 & 1313.1 & 1164.1 & -149 & 11.35 \\
\hline
\end{tabular}

\section{The modal analysis of the optimized lathe bed}

The 6 order natural frequency and vibration models of the integrated lathe bed are analyzed. Table 3 (d) shows the boundary conditions for modal analysis, and table 3 (e) (g) shows the 3 order vibration models.

The first three modal frequencies respectively are $371.87 \mathrm{~Hz}, 616.88 \mathrm{~Hz}$ and $706.89 \mathrm{~Hz}$, as far as possible to avoid the first three order vibration frequency.

\section{Conclusion}

Based on the solid isotropic material with penalization approach, the $\mathrm{CNC}$ lathe mathematical model of topology optimization is developed, in which minimization of the compliance is taken as objective function and the volume is taken as constraint function; Then got the best optimal topology form of the bed through the topology optimization, new geometric model was reconstructed according to the topology form. The analysis of primary and reconstruction models of the bed is shown as:

(1) The optimized lathe bed maximum deformation is reduced to $0.09 \mathrm{~mm}$ that is less than 0.29 $\mathrm{mm}$ of primitive structure so as to improve the static stiffness;

(2) Compared to the primitive structure, the quality of the optimized structure reduces almost $10 \%$, which make the body more save materials, reduce the manufacturing cost;

(3) The maximum stress of the optimized structure increases nearly $10 \mathrm{Mpa}$ that is far less than the tensile strength of $300 \mathrm{Mpa}$;

(4) Compared with the original bed the first 5 order modal frequency of optimized lathe bed diversely enhances so as to reduce the risk in the low frequency resonance, basically meet the design requirements.

\section{Acknowledgements}

This work was financially supported by Beijing areal Project of Ministry of Education (KM2016-1085-3003), the national natural science foundation of China (51275174) and the research subject of Beijing Polytechnic College in 2016 (bgzyky201617).

\section{References}

[1] Du Yi-xian, Yan Shuang-qiao ,etc.Static and dynamic multi-objective topology optimization design on bed of CNC gear shaper [J].Mechanical design, 2014, 31 (6): 64-67.

[2] Frecker M.I., Ananthasuresh G. K., Nishiwaki S.Topological synthesis of compliant 
mechanisms using multi-criteria optimization", Transactions of the ASME, Journal of Mechanical Design, 1997,119(2): 238-245.

[3] Nishiwaki S, Frecker M I, Min S, et al. Topology optimization of compliant mechanisms using homogenization method. International Journal for Numerical Method Engineering, 1998, 42 (3): 535-559.

[4] Li Zhao-kun, Zhang Xian-min. Reliability-based topology optimization of a compliant micro-gripper with geometrical nonlinearity, $[\mathrm{J}]$. Journal of south China university of technology (natural science edition),2008, 36 (8): 110-116.

[5] Zhang Xian-min. Topology optimization of compliant mechanism [J]. Journal of mechanical engineering, 2003, 39 (11): 47-51.

[6] Zhan Jin-qing, Zhang Xian-min. research on static and dynamic multi-objective topology optimization of continuum structures [J].Journal of Mechanical Strength, 2010，32 (6): 933-937.

[7] Sigmund, O. Design of Multiphysics Actuators using Topology Optimization-Part I : One-material Structures, Computer Methods in Applied Mechanics and Engineering, 2001, 190(49-50):6577-6604.

[8] Sigmund O. On the design of compliant mechanisms using topology optimization, Mechanics of Structures and Machines, 1997, 25(4):495-526. 